\title{
Large polarons in dry DNA: temperature and anharmonic effects
}

\author{
Z. Ivic ${ }^{1, a}$ and D. Toprek ${ }^{2}$ \\ ${ }^{1}$ University of Belgrade, "Vinča" Institute of Nuclear Sciences, Laboratory for Theoretical and \\ Condensed Matter Physics-020, P.O. Box 522, 11001 Belgrade, Serbia \\ ${ }^{2}$ University of Belgrade, "Vinča" Institute of Nuclear Sciences, Laboratory for Nuclear and Plasma \\ Physics-011, P.O. BOX 522, 11001, Belgrade, Serbia
}

\begin{abstract}
We study the influence of temperature on large polarons in DNA molecules. Employing the mean field approximation we derive the slight generalization of the SSH Hamiltonian with temperature dependent parameters. It was found that the degree of the modification of system parameters is very weak at physiologically relevant temperature which indicates that the free acoustic polarons as charge carriers may be responsible for DNA conductivity.
\end{abstract}

\section{Introduction}

Charge (electron, hole) migration in DNA molecule is the subject of great scientific and practical interest. In particular, hole (electron) transfer along DNA may be associated with damage, mutation and repair processes in DNA [1,2]. On the other side, possible large conductivity of DNA molecule implies its engineering application in nanotechnology - design of nanostructured devices and components in molecular electronics, for example. In spite of the intense investigations the mechanism of charge transfer in DNA is still controversial and subject of vivid debate for years $[1,2]$. Its elucidation on microscopic level would be essential for the understanding of the some basic phenomena in biochemistry and for further progress in DNA based molecular technologies and in molecular electronics.

Various mechanisms have been proposed as a possible explanation of the charge (electron, hole) motion along the DNA molecule but, without the definite resolution of the issue. During the last decade polaron mechanism has been actively considered as a possible mechanism of charge transfer in DNA [3-10]. Such an approach had certain success in the explanation of experimental data and the part of the existing controversies has been resolved within the polaron model. Nevertheless, it is still unclear whether the transport processes occur via the hopping of small polarons between adjacent sites or by means the coherent propagation of large polarons. In particular, both types of behaviours have been considered [3-10].

In this paper we address problem of the influence of the temperature on the large polaron properties. The temperature effects, so far, were mainly investigated numerically within the phenomenological approaches: the influence of thermal fluctuations was modelled by means of damping of phonon subsystem due to its coupling with phenomenological random forces [9]. Results

\footnotetext{
a e-mail : zivic@ vinca.rs
}

This is an Open Access article distributed under the terms of the Creative Commons Attribution License 2.0, which permits unrestricted use, distribution, and reproduction in any medium, provided the original work is properly cited. 
obtained within such an approach substantially depend on the particular choice of phenomenological parameters. Thus, in order to avoid such arbitrariness, we propose an alternative microscopic approach based on mean field approximation which enables one to take into account the nonlinear terms in lattice displacements.

\section{Effective Hamiltonian}

Although the realistic DNA structure is aperiodic, in some realistic situations long distance charge transfer takes place between two guanine-cytosine (G-C) pairs which are separated by many periodically arranged adenine-thymine (A-T) pairs. Therefore, charge migration within DNA may be described by the relatively simple tight binding models [12] in which the charge migration along the DNA stack is modelled in terms of on-site energies and nearest neighbouring overlap integrals. One of the simplest models which still captures the essentials physics of the charge transfer in DNA stack is specified in terms of the one dimensional model Hamiltonian

$$
H=-\frac{1}{2} \sum_{<f, g>1}^{N} J_{f, g} B_{f}^{+} B_{g}+\sum_{f=1}^{N} \frac{P_{f}^{2}}{2 M}+\frac{1}{2} \sum_{<f, g>=1}^{N} V_{f, g} .
$$

The first term in above expression represent the usual tight binding Hamiltonian of the electronic subsystem, while the remaining ones correspond to the Hamiltonian of the lattice modes. Here the each individual site, labelled here as $\boldsymbol{f}(\boldsymbol{g})$, represents a base pair. Symbol $\langle\boldsymbol{f}, \boldsymbol{g}\rangle$ is used to denote that summation runs over the nearest neighbours, while the factor $1 / 2$ is included in order to avoid the double summation over the repeated indices. $\boldsymbol{J}_{\boldsymbol{f}, \boldsymbol{g}}$ denotes the overlap integral of electron (hole) hopping between nearest neighbouring sites and satisfy $\boldsymbol{J}_{f, g}=\boldsymbol{J}_{g f}$. Operator, $\boldsymbol{B}_{f}^{+}\left(\boldsymbol{B}_{f}\right)$ creates (annihilates) electron (hole) on each base par. The electron spin is not essential in the context of the single charge transport and will be disregarded hereafter. Parameters of the lattice subsystem are: $\boldsymbol{M}$ the mass of A-T base pair $\left(M \approx 4.35 \cdot 10^{-35} \mathrm{~kg}\right)$, its momentum $\boldsymbol{P}_{f}$ and the lattice potential energy $\boldsymbol{V}_{\boldsymbol{f}, \boldsymbol{g}}$. Finally, $N \gg>1$ represents the total number of the A-T pairs that participates in transfer.

The dynamics of lattice induces the base pair displacement from their equilibrium position which we formally account for as follows: $\boldsymbol{f} \rightarrow \boldsymbol{f}+\boldsymbol{u}_{f}$, where $\boldsymbol{u}_{f}$ represents the longitudinal displacement of $\boldsymbol{f}$ base from its equilibrium position. The influence of lattice dynamics may be now formally accounted by expanding the energy parameters in (1) in powers of $\boldsymbol{u}_{f}$ which is considered to be small in respect to the equilibrium inter-base separation. The usual treatment is based upon the harmonic approximation, thus the resulting model Hamiltonian is derived keeping only the terms of the lowest order in $\boldsymbol{u}_{f}$-linear in electron-phonon interaction and quadratic in lattice potential energy. This implicitly assumes that there is no thermal expansion, that lattice elastic constants are independent of pressure and temperature, etc. The accounting of these effects is necessary for the proper description of the vibrational subsystem at finite temperatures in soft, easy deformable, biological macromolecules such DNA. For that purpose we apply the mean field procedure and derive the effective Hamiltonian with temperature dependent energy parameters. In the first step we exploit the lattice periodicity and expand the energy parameters of system in Fourier series. In such a way, the electronic overlap integral becomes

$$
J_{f, g}=\frac{1}{\sqrt{N}} \sum_{k} J_{k} e^{i k a(f-g)} e^{i k\left(u_{f}-u_{g}\right)} \equiv \frac{1}{\sqrt{N}} \sum_{k} J_{k} e^{i k a(f-g)}\left[\cos k a\left(u_{f}-u_{g}\right)+i \sin k a\left(u_{f}-u_{g}\right)\right]
$$

Here $J_{k}=\left(1 / N^{1 / 2}\right) \Sigma_{f} J_{f} e^{i k f a}$ and becomes $J_{k}=(2 / N)^{1 / 2} J \cos k a$ in the nearest neighbour approximation. Here $\boldsymbol{J}$ stands for the overlap integral between nearest neighbouring sites; $a$ is the lattice spacing and $k$ is the wave vector that runs from $-\pi / a$ to $\pi / a$. Trigonometric form in (2) is convenient for the practical calculations of the average values. Now we proceed in a mean field manner and simply replace nonlinear terms in lattice displacements by the corresponding average values. In such a way the first term in (2) becomes $\cos k a\left(u_{f}-u_{g}\right) \rightarrow\left\langle\cos k a\left(u_{f}-u_{g}\right)\right\rangle_{0}$. Note that the same procedure would give vanishing result for the second term. For that reason we first extract the terms linear in lattice displacements and then, performing the averaging in final step, we obtain: 


$$
\sin k a\left(u_{f}-u_{g}\right)=2 \sin \frac{k a}{2}\left(u_{f}-u_{g}\right) \cos \frac{k a}{2}\left(u_{f}-u_{g}\right) \rightarrow k a\left(u_{f}-u_{g}\right)\left\langle\cos \frac{k a}{2}\left(u_{f}-u_{g}\right)\right\rangle_{0} .
$$

Here $\langle\ldots\rangle_{0}$ denotes the averaging over the harmonic phonon distribution. Formation of large polaron appears in the adiabatic regime when electron bandwidth highly exceeds maximal phonon energy. Therefore, the vibrational subsystem is slow as compared with the electronic and may be treated as a classical one. Thus, the aforementioned averaging is performed over the classical phonon distribution:

$$
\langle\ldots\rangle_{0}=\frac{\prod_{f} \int d P_{f} \int d u_{f} \ldots e^{-\frac{H_{0}}{\theta}}}{\prod_{f} \int d P_{f} \int d u_{f} e^{-\frac{H_{0}}{\theta}}}, H_{0}=\sum_{f} \frac{P_{f}^{2}}{2 M}+\frac{\kappa}{2} \sum_{f}\left(u_{f+1}-u_{f}\right)^{2} .
$$

Here $\boldsymbol{H}_{\mathrm{o}}$ stands for the lattice Hamiltonian in the harmonic approximation. This, finally yields

$$
\begin{aligned}
& H_{M F}=-J(\theta) \sum_{f=1}^{N}\left(B_{f}^{+} B_{f+1}+h . c\right)+\alpha(\theta) \sum_{f=1}^{N}\left(B_{f}^{+} B_{f+1}+h . c\right)\left(u_{f+1}-u_{f}\right)+ \\
& \sum_{f=1}^{N} \frac{P_{f}^{2}}{2 M}+\frac{\kappa(\theta)}{2} \sum_{f=1}^{N}\left(u_{f}-u_{f+1}\right)^{2} .
\end{aligned}
$$

Here, $J(\theta)=\frac{J}{N} \sum_{k} e^{-2 \frac{\theta}{\theta_{D}} a^{2} k^{2}} \cos ^{2} k a, \alpha(\theta)=\frac{J}{2 N} \sum_{k} \frac{k}{a} e^{-\frac{\theta}{2 \theta_{D} a^{2} k^{2}}} \sin 2 k a$ and

$\kappa(\theta)=\frac{4 \kappa}{N} \sum_{k} k^{2} e^{-\frac{\theta}{4 \theta_{D}} a^{2} k^{2}} \cosh ^{2}\left(\frac{\theta a^{2} k^{2}}{4 \theta_{D}}\right) \cos ^{2} k a$ represent the effective, temperature renormalized,

overlap integral, electron-phonon coupling constant and elastic constant, respectively. Here $\theta=k_{B} T$, denotes temperature in energy units, while $\theta_{D}=\kappa a^{2}$ stands for the characteristic phonon temperature.

\section{Results and discussions}

The above model Hamiltonian is formally identical to that of the famous Su, Schriefer, Heeger (SSH) model [13] which has been recently proposed as a theoretical background for the studies of the migration of excess charges, electron or hole, in DNA [5-9,11]. However, in comparison with previous studies the energy parameters in our model Hamiltonian are temperature dependent which enables the study of the impact of temperature on large polaron properties. For that purpose it is necessary to evaluate the afore-derived energy parameters which demand the evaluation of the sums over the wave vectors $\boldsymbol{k}$. Practical calculation has been performed numerically after the substitution of the summation over $\boldsymbol{k}$ by the integration in accordance with the rule- $\Sigma_{k} \ldots \rightarrow \frac{\pi}{2 a} \int_{-\pi / a}^{\pi / a} \ldots . d k$.

Temperature induced modifications of system parameters are presented in Fig. 1.

It turns out that the temperature induced changes of system parameters are very weak and range up to $5 \%$ on $300 \mathrm{~K}$. This is the consequence of the fact that characteristic temperature $\theta_{D}$ for DNA, as estimated on the basis of the measurements of speed of sound in DNA as $1.9 \mathrm{~km} / \mathrm{s}\left(\kappa=0.87 \mathrm{eV} / \AA^{2}\right)$ [5-9], is far above the physiologically relevant temperatures $\left(\mathrm{T}_{\mathrm{D}} \sim 10^{5} \mathrm{~K}\right)$. This means that, in physiologically the most interesting interval, temperature only slightly modifies system parameters and cannot affect polaron substantially. In particular, its size, being proportional to $\boldsymbol{J}(\boldsymbol{\theta})$, decreases up to $5 \%$, while, in the same time, its binding energy $\left(\sim \alpha^{4}(\theta)\right)$ may decrease up to $8-10 \%$. That is, large polaron in DNA is robust entity and thermal fluctuations can not substantially affect transport properties of DNA provided that charge transport is achieved through the motion of large polarons. 


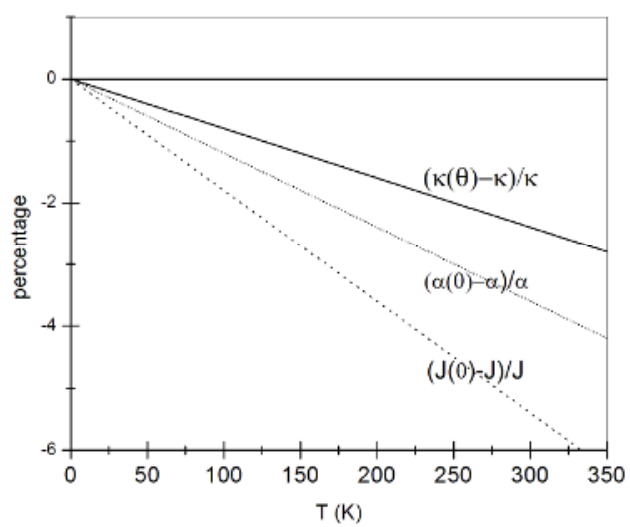

Fig. 1. The relative changes of the system parameters versus temperature.

\section{Acknowledgment}

This work was supported by the Serbian Ministry of Science and Technology: under the contract numbers III-45010 and OI-171009.

\section{References}

1. C. Dekker, M.A. Ratner, Phys. World 14, 29 (2001)

2. G. B. Schuster, Ed. Long-Range Charge transfer in DNA I, Top. Curr. Chem. 236, (2004)

3. G. B. Schuster, Ed. Long-Range Charge transfer in DNA II, Top. Curr. Chem. 237, (2004)

4. M. A. Ratner and J. Jortner, Molecular Electronics (Blackwell, Oxford, 1997).

5. E. M. Conwell, S.V. Rakhmanova, Proc. Natl. Acad. Sci. (USA) 97, 4556 (2000)

6. E. M. Conwel, Proc. Natl. Acad. Sci. 102, 8795 (2005)

7. E. Conwell, Top. Curr. Chem. 237, 73 (2004)

8. E. B.Starikov, Philos.Mag. 85, 3435 (2005)

9. E. M.Conwell, D.M.Basko, J. Phys. Chem. B110, 23603 (2006)

10. J. ortner, M.Bixon, T.Langenbacher, M.E.Michel-Beyerle, Proc. Natl. Acad. Sci. (USA) 95, 12759 (1998)

11. C. M. Changa, A.H.Castro Neto, A.R.Bishop, Chem.Phys. 303, 189 (2004)

12. S. S. Mallajosyula, S. K.Pati, J.Phys.Chem.Lett. 1, 1881 (2010)

13. W. P. Su, R. Schrieffer, A. Heeger, Phys. Rev. B22, 2099 (1980). 